\title{
A positive feedback-based gene circuit to increase the production of a membrane protein
}

\author{
Karan Bansal' , Ke Yang², Goutam J Nistala³ ${ }^{3}$ Robert B Gennis² and Kaustubh D Bhalerao*3
}

\begin{abstract}
Background: Membrane proteins are an important class of proteins, playing a key role in many biological processes, and are a promising target in pharmaceutical development. However, membrane proteins are often difficult to produce in large quantities for the purpose of crystallographic or biochemical analyses.
\end{abstract}

Results: In this paper, we demonstrate that synthetic gene circuits designed specifically to overexpress certain genes can be applied to manipulate the expression kinetics of a model membrane protein, cytochrome bd quinol oxidase in E. coli, resulting in increased expression rates. The synthetic circuit involved is an engineered, autoinducer-independent variant of the lux operon activator LuxR from V. fischeri in an autoregulatory, positive feedback configuration.

Conclusions: Our proof-of-concept experiments indicate a statistically significant increase in the rate of production of the $b d$ oxidase membrane protein. Synthetic gene networks provide a feasible solution for the problem of membrane protein production.

\section{Background}

One of the goals of the emerging discipline of synthetic biology is to make it easy to 'reprogram' the behavior of living organisms by transforming them with novel, rationally engineered, synthetic gene networks. Engineered gene networks have numerous applications in diverse biotechnological endeavors such as metabolic engineering [1,2], single cell biosensors [3,4] as well as cell-based computers [5,6]. Precise regulation of gene expression either through exogenous control or through endogenous autoregulation is often critical for the development of synthetic biology applications. Several strategies have been developed for gene regulation including the development of libraries with modified promoter sequences [7], development of gene expression toggle switches controlled by exogenous inputs [8], gene expression pulse generators [9] and oscillators [10], and even post-transcriptional regulatory systems [11]. There are numerous reviews detailing the engineering principles behind the development of synthetic gene circuits, recent advances, and potential applications in the future [12-15].

* Correspondence: bhalerao@illinois.edu

${ }^{3}$ Department of Agricultural and Biological Engineering, University of Illinois at Urbana Champaign, 1304 W. Pennsylvania Ave, Urbana, IL 61801 USA Full list of author information is available at the end of the article
Autoregulation by way of positive or negative feedback is commonly used as a motif in gene regulatory circuits. Depending on the specific circuit topology, feedback often leads to improved performance of the gene regulatory circuits. Specifically, a positive feedback within the regulatory circuit can lead to increased steady state level (amplification) of the gene expression, faster response kinetics, and increased sensitivity to exogenous inducers or autoinducers [16-19]. These properties are particularly suitable for applications such as protein production, where the benefits of increased production rates can directly translate into reduced resource costs and increased profits.

While gene circuits are routinely used to express soluble proteins such as green fluorescent protein (GFP) in model circuits, they have not been applied for the production of membrane proteins. The structure and biochemistry of membrane proteins are extremely important to study. About $20 \%$ of all genomes sequenced so far appear to encode membrane proteins [20]. Additionally, their key role in the etiology of many diseases make them targets for therapeutic intervention. $60-70 \%$ of all drug targets are membrane proteins, such as the G-protein coupled receptor family [21,22].

Membrane proteins are very difficult to produce [22]. They are normally expressed at very low levels in nature. 
It is possible to express them at higher levels using strong promoters such as the hybrid tac and trc promoters, or high-activity polymerases such as the T7 RNA polymerase, however there are some inherent drawbacks with these methods such as leaky expression or promoter toxicity. Additionally, heterologous expression of membrane proteins can cause aggregation of the product within the cells. These inclusion bodies have to be separated and refolded in order to reconstitute their native structure. This is often an inefficient and laborious process. We note that there are recent developments in the field of membrane protein expression, such as the development of tunable T7 RNA polymerase-based systems (the so called "Walker strains", C41(DE3) and C43(DE3)) that are not significantly affected by T7 RNAP toxicity [23]. Positive feedback-based synthetic gene circuits offer an alternative to the gene expression techniques discussed above. In this study, we explored the possibility of increasing the expression of the model membrane protein, cytochrome $b d$ quinol oxidase (also referred to as $b d$ oxidase), a terminal oxidase from $E$. coli. Normally, $b d$ oxidase is expressed under microaerobic conditions since it has a high affinity to oxygen and serves as an oxygen scavenger in low oxygen conditions $[24,25]$. Further, the biochemistry of $b d$ oxidase is well-characterized, and methods to measure the gene product levels spectrophotometrically are well established [26]. These features make $b d$ oxidase a good test case to explore the positive feedback-based gene expression system.

\section{Materials and methods}

\section{Design of the synthetic gene network}

A positive feedback circuit was built using elements from the quorum sensing system in Vibrio fischeri and the $b d$ oxidase gene as shown in Figure 1. LuxR is a transcriptional activator from the $V$. fischeri quorum sensing system that binds to its cognate promoter $p_{l u x}$ and activates expression of genes under its control. The wild type LuxR is inactive when produced. Acyl homoserine lactone (AHL), produced by another gene, luxI, is an autoinducer that binds LuxR and increases its activity. There are other

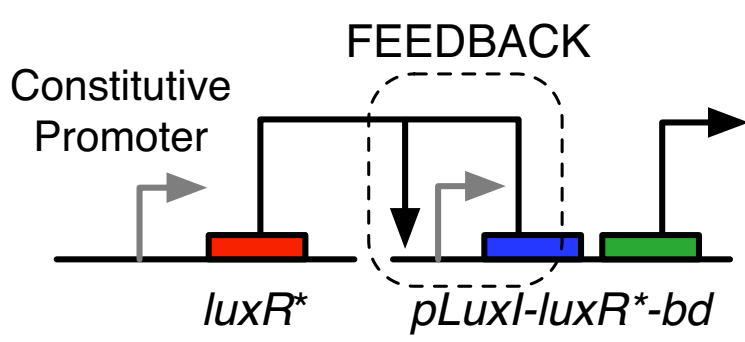

Figure 1 A schematic showing the positive feedback-based gene expression system. similar positive feedback circuits based on the wild type LuxR, which rely on the action of AHL, either exogenously supplied, or produced by the action of luxI, for their expression system to operate correctly.

In our circuit, we specifically desired a regulatory protein that was not reliant on any external co-factors, and operated independent of the cell density in the culture. Fortunately, the structure-activity relationship of LuxR is well-known. Previous studies have determined that an Ala221TVal mutant of LuxR (denoted LuxR*) activates the $p_{\text {lux }}$ promoter even in the absence of AHL $[19,27]$.

The gene network design consisted of two plasmids. The first plasmid encoded a copy of the $l u x R^{*}$ gene under a constitutive promoter. The second plasmid encoded a copy of the $b d$ oxidase gene, along with another copy of the luxR* gene in a bi-cistronic configuration. The twoplasmid system was chosen to ensure modularity in design and to enable the facile construction of experimental controls to validate the positive feedback.

\section{Selection of the host strain}

E. coli strain $M L 15 A$ is a $b d$ oxidase knockout derivative of the "Walker strain" C43 (DE3) (F-ompT hsdS $S_{B}$ $\left.\left(r_{B}^{-} m_{B}^{-}\right) g a l d c m c y d^{-}\right)$, and was used as the host strain for expressing the wild-type $b d$ oxidase from a plasmid. It has a tendency to overproduce membrane proteins [23]. All cloning work was performed by electroporation using electrocompetent ML15A cells. The C43 (DE3) wild type control does not have any plasmids, and has a chromosomal $b d$ oxidase gene, and was used as a control for observing native expression of $b d$ oxidase.

\section{Plasmid construction}

Plasmids pGN3, pKB1 and pKB2 were based on the $\mathrm{PRO}^{\text {ra }}$ Tet6xHN bacterial expression system (Clontech Laboratories, Mountain View, CA). The PRO ${ }^{\mathrm{ma}}$ Tet6xHN plasmid formed the basis of the plasmids constructed for this experiment, which are described below.

\section{Constitutive plasmid}

The ColE1 origin from PRO ${ }^{\mathrm{m}} \mathrm{Tet} 6 \mathrm{xHN}$ was replaced by p15A between the restriction sites $\mathrm{XbaI}$ and SacI as described by Lutz and Bujard [28]. The chloramphenicol resistance marker was replaced by the ampicillin resistance marker $\left(\mathrm{Ap}^{r}\right)$ from pZE21 between the unique restriction enzyme sites XhoI and SacI yielding pPROTetE-Kan-p15A.

The luxR* gene was first amplified using primers KW78F1 [AAC TTT ATA AGG AGG AAA AAC ATA TGA AAA ACA TAA ATG CCG AC] and KW078R [ACT GTC GAC TTA ATT TTT AAA GTA TGG GC]. 
The resulting product was then amplified using primers KW078F2 [TAT GAA TTC AAC TAA AGA TTA ACT TTA TAA GGA GGA AAA ACA ] and KW078R to introduce the EcoRI cut site (shown in boldface). This product was then digested with EcoRI and SalI and sub-cloned into the EcoRI and SalI cut-sites of pPROTetE-Kan-p15A. KW079F [ATA GGT CTC TGT GCA AAT GAA ACT CAA TAC AAC] and KW079R [ATA GGT CTC TGC ACA TTG GTT AAA TGG AAA GTG A] were then used to perform a whole plasmid PCR on the above to introduce a mutation GCGTGTG (Ala221TVal) in luxR. The resulting PCR product was then digested and blunt end ligated with BsaI to obtain pGN3. The plasmid pGN3 thus consisted of the p15A origin of replication and the ampicillin resistance marker $\left(\mathrm{Ap}^{r}\right)$ (Figure 2). It consists of a copy of the $l u x R^{*}$ gene under the control of the promoter $p_{\text {LtetO-1. }}$. In the presence of the repressor TetR, this promoter is tightly repressed, and can be induced by the addition of anhydrotetracycline (aTc). However, the $M L 15 A$ host strain used in this experiment does not produce

TetR, and thus the $l u x R^{*}$ gene is expressed constitutively in the host. The pGN3 plasmid was used as the constitutive plasmid (CP).

\section{Feedback plasmid}

pGN29 consists of a plasmid harboring the ColE1 origin of replication, chloramphenicol resistance $\left(\mathrm{Cm}^{r}\right)$, a green fluorescent protein $(g f p)$ and a copy of lux $R^{*}$ under the control of the lux promoter $\left(p_{l u x}\right)$. The $g f p$ gene was replaced by the $b d$ oxidase gene producing the plasmid $\mathrm{pKB} 1$. The $b d$ oxidase gene was extracted from the pTY1 plasmid by PCR using the forward primer 5'(ATA GAA TTC GCG ATG AGC AAG GAG TCA TG ATG TTA

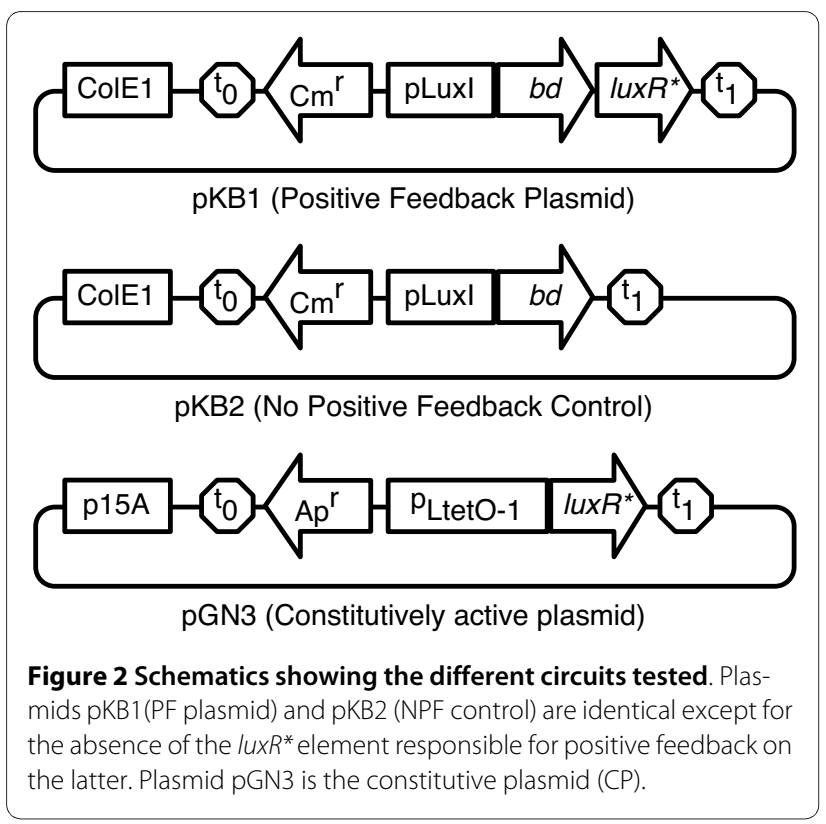

GAT ATA GTC G)3' and the reverse primer 5'(ATT AAG CTT CGC TTA GTA CAG AGA GTG GGT GTT ACG TTC AAT ATC)3'. The forward primer contained an EcoRI site (shown in boldface) and the reverse primer contained HindIII site. pGN29 and the PCR product were digested by using EcoRI and HindIII. The PCR product obtained was ligated with the digested pGN29 to obtain the positive feedback plasmid pKB1. The resulting $5.5 \mathrm{~kb}$ plasmid was separated using gel electrophoresis.

\section{No positive feedback control plasmid}

Plasmid pGN23 consists of a ColE1 origin of replication, chloramphenicol resistance and $g f p$ similar to pGN29. However it contains a $p_{\text {LtetO-1 }}$ promoter, which was replaced by the $p_{l u x}$ promoter. In addition, it does not contain the $\operatorname{lux} R^{*}$ gene. The $b d$ oxidase region was extracted from pTY1 using the same procedure as above, and was similarly ligated with the pGN23 plasmid to obtain the no positive feedback control plasmid pKB2. The resulting plasmid was $4.7 \mathrm{~kb}$.

All plasmids were verified using DNA sequencing. The plasmid maps for pKB1, pKB2 and pGN3 are shown in Figure 2. Table 1 shows a list of treatments and various controls along with their designations.

\section{Growth media}

For all experiments, cells were grown in $5 \mathrm{ml}$ of LB medium (BD Bacto ${ }^{\mathrm{m}}$ Tryptone, BD Bacto ${ }^{\mathrm{m}}$ Yeast extract, BD Bacto ${ }^{\mathrm{m}}$ agar, sodium chloride) with appropriate antibiotics (Sigma Aldrich, St. Louis, MO). Depending on the plasmid's antibiotic resistance, a combination of $100 \mu \mathrm{g} /$ $\mathrm{ml}$ ampicillin and $34 \mu \mathrm{g} / \mathrm{ml}$ chloramphenicol was used.

\section{Preparation of inoculum}

The host strain with the appropriate plasmids was grown initially in $5 \mathrm{ml}$ culture tubes at $30^{\circ} \mathrm{C}$ in Gyrotory water bath shaker (Model G76, New Brunswick Scientific, Edison, NJ) overnight in LB medium with the appropriate antibiotics. $2 \mathrm{ml}$ of the overnight culture was transferred to $200 \mathrm{ml}$ of fresh LB medium in $500 \mathrm{ml}$ shake flasks with the appropriate antibiotics and grown at $30^{\circ} \mathrm{C}$ for $10-12$ hours, until the culture reached an optical density of about 0.5 .

\section{Scale up and sampling}

$15 \mathrm{ml}$ of the inoculum prepared as above was transferred to each of the twenty four $2 \mathrm{l}$ flasks containing $1 \mathrm{l} \mathrm{LB}$ medium with the appropriate antibiotics. These flasks were shaken at $220 \mathrm{rpm}$ at $30^{\circ} \mathrm{C}$ using an Innova 4330 incubator shaker (New Brunswick Scientific, Edison, NJ). Samples were taken at 4, 8, 10, 12, 16 and $20 \mathrm{~h}$. At each sampling time four 21 flasks were removed from the shaker and the cells were peletted at $11,220 \times g$ for 20 $\mathrm{min}$. The pellet was stored at $-80^{\circ} \mathrm{C}$ until further use. 
Table 1: Experimental design to test the effect of the positive feedback.

\begin{tabular}{lll}
\hline Circuits tested & Key & Remarks \\
\hline pKB1+pGN3 & PF $+C P$ & Treatment \\
pKB2 + pGN3 & NPF $+C P$ & Control to test the effect of positive feedback \\
pKB2 & NPF & Control to test the leakiness of the $p_{\text {lux }}$ promoter \\
pKB1 & PF & Control to test the combined effect of the leakiness of the promoter and the positive feedback \\
N/A & WT & Control to test wild type expression levels \\
\hline
\end{tabular}

\section{Protein sample preparation}

The cell pellets were removed from $-80^{\circ} \mathrm{C}$ and suspended in $100 \mathrm{ml}$ of cell lysis buffer (100 mM Tris, $15 \mathrm{mM}$ ethylenediaminetetraacetic acid (EDTA), $15 \mathrm{mM}$ Benzamidine, $\mathrm{pH}$ 8.3). Deoxyribonuclease I (Sigma Aldrich, St. Louis, MO) and protease inhibitor cocktail (P8465, Sigma Aldrich, St. Louis, MO) were also added to the above suspension. After the sample was completely suspended in the buffer, cells were broken down by passing the sample 6 times through a nitrogen decompression based cell disruptor. The sample collected after disruption was then centrifuged at $11,220 \times g$ for $20 \mathrm{~min}$ and the pellet was discarded. The supernatant was spun down in an ultracentrifuge (L7-65, Beckman Coulter, Fullerton, CA) at $125,000 \times g$ for $4 \mathrm{~h}$. The pellets were collected and stored at $-80^{\circ} \mathrm{C}$ until further processing. The membranes collected for the different time intervals were then taken out from the $-80^{\circ} \mathrm{C}$ freezer and $0.5 \mathrm{~g}$ of each sample was weighed out and solublized in $30 \mathrm{ml}$ of buffer $(50 \mathrm{mM}$ monopotassium phosphate, $25 \mathrm{mM}$ potassium chloride, 5 $\mathrm{mM}$ ethylenediaminetetraacetic acid (EDTA), $0.75 \%$ sucrose monododecanoate $\mathrm{w} / \mathrm{v}, \mathrm{pH}$ 6.5). The suspended sample was spun down in an ultracentrifuge at 125,000 $\times$ $g$ for $2 \mathrm{~h}$ and the supernatant was collected. The supernatant was used to find the concentration of heme $d$ in each sample by obtaining the absorbance spectrum of the sample.

\section{Spectroscopic measurements}

All of the absorbance spectra in the UV-Visible region were obtained with a UV-Visible spectrophotometer (8453, Agilent Technologies, Santa Clara, CA) using a 1$\mathrm{cm}$ path-length cuvette. For each sample, absorption spectra were obtained for both air oxidized and reduced heme $d$. The sample was reduced by adding sodium dithionite in excess. Cytochrome bd oxidase has a chlorin chromophore (heme D) exhibiting a characteristic absorption maximum at $628 \mathrm{~nm}$ in the fully reduced state. The bd oxidase concentration was determined from the difference in the absorption spectra between dithionite-reduced and air-oxidized bd oxidase levels using the following equation [29]:

$$
\Delta_{628-607 n m}^{2}=10.8 \mathrm{mM}^{-1} \mathrm{~cm}^{-1}
$$

In this way, one can quantify the amount of active bd oxidase in the membrane. The absorption at $607 \mathrm{~nm}$ is not affected by bd oxidase and serves to eliminate any artifacts due to baseline shifts in the absorbance spectrum.

\section{Results and Discussion}

Table 2 shows the results of the experiments. Each data point corresponds to one reading of the level of bd oxidase pooled from four 11 culture flasks. All gene circuits tested show bd oxidase levels that eventually rise above the background expression level of the wild type. It is expected that some of the cultures show bd oxidase levels lower than wild type initially, because unlike the wild type, the $M L 15 A$ host strain is a bd oxidase knock out. The wild type had an average concentration of 0.68 ( \pm $0.31) \mu M$ of bd oxidase as compared to $3.05( \pm 0.14) \mu M$ for the two-plasmid systems. In addition, positive feedback has a significant effect on the kinetics of expression of $b d$ oxidase. Figure 3 shows the graph of $b d$ oxidase expression. In order to determine the statistical significance of the results, the following form of the logistic growth model was fitted to the data:

$$
[b d]=\frac{A}{1+e \frac{d-t}{s}}
$$

Here, [bd] is the concentration of bd oxidase in $\mu M, A$ is the final asymptotic level of the growth curve, $d$ is the time required for half-maximal expression, $s$ is a scaling parameter and $t$ is time in hours. This model captures all the parameters of interest of the positive feedback, including amplification $(A)$, delay in expression $(d)$, and system response kinetics $(s)$. For the sample size of six data points per curve, the model parameters in Equation 2 were computed using a non-linear fitting function pro- 
Table 2: Concentration in micromoles $(\mu M)$ of bd oxidase produced by the various gene circuits.

\begin{tabular}{cccccc}
\hline $\begin{array}{c}\text { Time } \\
\text { (hrs) }\end{array}$ & $\begin{array}{c}\text { Treatment } \\
\text { PF+CP }\end{array}$ & NPF+CP & PF onlY & NPF only & WT \\
\hline 4 & 1.050 & 0.663 & 0.487 & 0.173 & 0.483 \\
8 & 2.838 & 1.354 & 0.601 & 0.101 & 0.715 \\
10 & 3.114 & 1.670 & 0.537 & 0.411 & 0.582 \\
12 & 2.983 & 1.848 & 1.040 & 1.184 & 0.655 \\
16 & 2.766 & 2.154 & 1.443 & 1.274 & 0.932 \\
20 & 3.052 & 2.701 & 1.627 & 1.373 & 0.763 \\
\hline
\end{tabular}

Refer Table 1 for a description of the treatment and controls.

vided by the open source statistical programming language $\mathrm{R}^{\circ}$ http://r-project.org. Table 3 shows the values of these parameters along with the statistics signifying the goodness-of-fit. Figure 4 shows the statistical comparison between the parameter values for the different circuits.

From the data and the computed model parameters (Figures 3 and 4), it is clear that positive feedback has a significant effect on the production kinetics of bd oxidase. This behavior has been previously reported by several researchers in literature for various cytosolic and

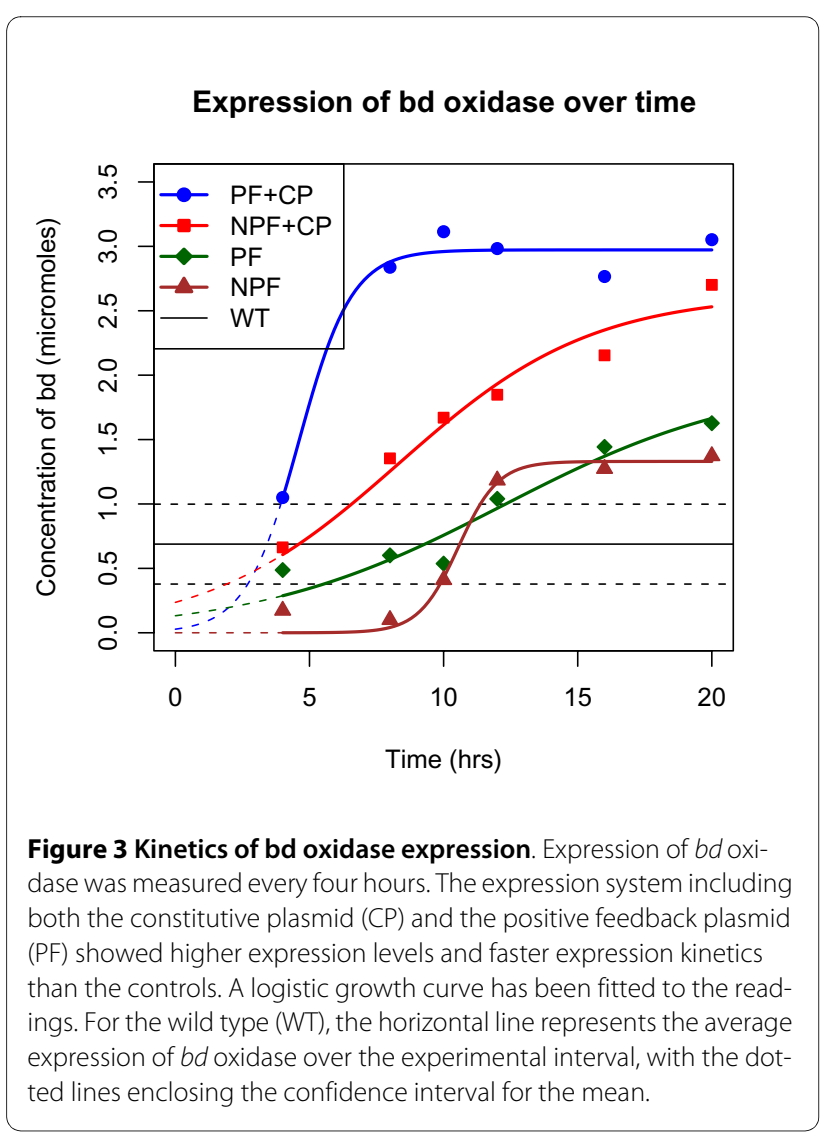

indicator proteins, and we confirm that it translates to the expression of membrane proteins as well. Qualitatively, our result is slightly different from previous work because in this case, there is a steric limit on the amount of membrane protein that can be produced, governed by the amount of membrane itself. Since the asymptotic membrane protein level is naturally capped, the membrane localization kinetics appear to speed up.

Ideally, one would need replicates to determine the variability from sample to sample for each of the data points. While replicates for each time point would have led to greater confidence in the actual values of the model parameters, as a series of six time points they are sufficient to show that there exist significant statistical differences between the treatment and the various controls. Moreover, the non-availability of the time points is a result of the original problem: A large culture volume is needed to produce a sample of membrane protein. In our case, each time point is a pooled sample of four $11 \mathrm{sam}-$ ples which leads to one measurable reading.

Nevertheless, within reason, certain conclusions can be drawn. Firstly, all circuits tested produced more bd oxidase than the wild type. This result was expected since $b d$ oxidase is typically expressed in microaerobic conditions, whereas the conditions in our experiment were aerobic. Secondly, all plasmids used were medium copy plasmids, and by virtue of having multiple copies of the same gene, one can expect a production level higher than that seen in the wild type. Thirdly, the constitutive plasmid (i.e. the constitutive promoter) plays a greater role in the final level of gene expression rather than the positive feedback, since there is no significant difference between the asymptote parameter $(A)$ of the treatment $(\mathrm{PF}+\mathrm{CP})$ and the NPF+CP control, while there is a significant difference between the NPF+CP and the NPF controls. Finally, the $p_{l u x}$ promoter, which is known to be leaky can cause undesirable expression of the membrane protein, which can lead to high expression levels if there is a positive feedback available to amplify this leaky expression. This is an important consideration when expressing toxic pro- 
Table 3: Logistic growth model parameters for the experimental data.

Asymptote $(A)$

\begin{tabular}{cccc}
\hline Circuit & Estimate & Std. Error & p-value \\
\hline PF+CP & 2.9724 & 0.0722 & $2.08 \mathrm{e}-06$ \\
NPF+CP & 2.6296 & 0.2365 & 0.000372 \\
PF & 1.9712 & 0.5349 & 0.0211 \\
NPF & 1.33029 & 0.06984 & $4.48 \mathrm{e}-05$ \\
WT* & 0.68 & 0.155 & $2.9 \mathrm{e}-06$ \\
\hline
\end{tabular}

Delay $(d)$

\begin{tabular}{cccc}
\hline Circuit & Estimate & Std. Error & p-value \\
\hline PF+CP & 4.6043 & 0.3199 & 0.000135 \\
NPF+CP & 8.3328 & 1.0092 & 0.001174 \\
PF & 12.182 & 3.235 & 0.0197 \\
NPF & 10.55178 & 0.26399 & $2.34 \mathrm{e}-06$ \\
\hline
\end{tabular}

Scale (s)

\begin{tabular}{cccc}
\hline Circuit & Estimate & Std. Error & p-value \\
\hline PF+CP & 0.9892 & 0.4051 & 0.071 \\
NPF+CP & 3.5978 & 0.8644 & 0.0141 \\
PF & 4.6276 & 1.8552 & 0.0672 \\
NPF & 0.73923 & 0.21563 & 0.0266 \\
\hline
\end{tabular}

For the WT, the average value was assumed to be the asymptotic value $(A)$, while the delay and scale parameters were not estimated.

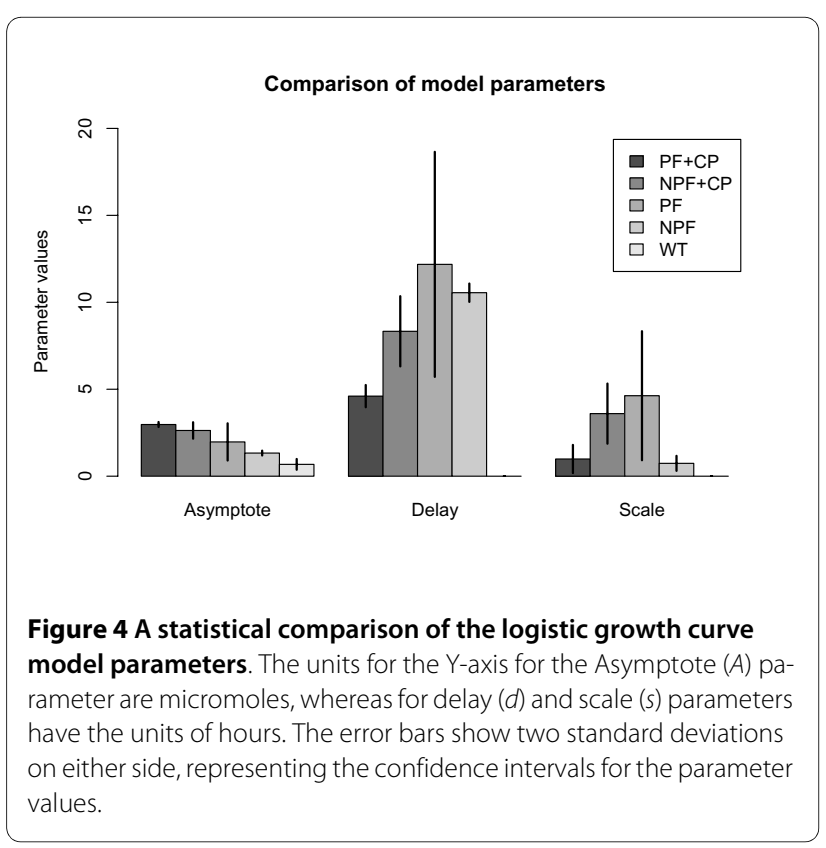

teins. The positive feedback really shows its benefits in reducing the delay in producing the protein. In other words, the positive feedback can reduce the time required to saturate a membrane with membrane protein, when viewed in comparison with the $\mathrm{NPF}+\mathrm{CP}$ control. However, the positive feedback might not be sufficient by itself and requires a driver plasmid for maximum productivity.

The scaling parameter $(s)$ gives an idea of the rate at which a positive feedback can enable a circuit to switch from the off state to the on state. As the scaling parameter tends to zero, the switching behavior looks more 'digital', i.e. with instantaneous switching between states. In this case, the parameter values are difficult to interpret. The treatment seems to have a high switching speed (low scaling parameter value), but at the same time the NPF control also seems to have a similar behavior, which could be a computational artifact due to the low final expression level of $b d$ oxidase in this case. 


\section{Conclusions}

One of the significant outcomes of the positive feedback expression system is that the time required to reach steady state levels of bd oxidase membrane protein reduces from $20 \mathrm{~h}$ (in the no feedback control case) to about $8 \mathrm{~h}$. There are, of course, several other factors that determine the precise numerical values of this performance gain, including promoter strength, plasmid copy number and the specific strain used. While these experiments do not have sufficient precision to quantify these performance gains, they do show that the positive feedback-based system accrues statistically significant gains.

One of the goals of synthetic biology is to develop reusable gene regulatory systems. The positive feedbackbased system we have developed is modular and can be readily extended as a building block in other protein expression systems. It can also be used to enhance existing protein expression systems, which use inducible promoters or exogenous polymerases such as T7 RNAP. It is therefore an attractive alternative to increase the yields of difficult-to-produce proteins such as membrane proteins.

\section{Competing interests}

The authors declare that they have no competing interests.

\section{Authors' contributions}

KDB and RBG conceived the experiments. KB did the experiments and wrote the manuscript draft. GJN helped with plasmid construction and with manuscript revision. KY helped with the experimental protocols. KB and KDB analyzed the data. All authors read and approved the final manuscript.

\section{Acknowledgements}

The authors would like to thank The University of Illinois for startup funds provided to KDB. The authors also acknowledge materials provided by Dr. Christopher V. Rao, Department of Chemical and Biomolecular Engineering at University of Illinois.

\section{Author Details}

'Dow AgroSciences, Indianapolis, IN, USA, ${ }^{2}$ Department of Biochemistry, University of Illinois at Urbana Champaign, USA and ${ }^{3}$ Department of Agricultural and Biological Engineering, University of Illinois at Urbana Champaign, 1304 W. Pennsylvania Ave, Urbana, IL 61801 USA

Received: 7 February 2010 Accepted: 25 May 2010

Published: 25 May 2010

\section{References}

1. Lee SK, Chou H, Ham TS, Lee TS, Keasling JD: Metabolic engineering of microorganisms for biofuels production: from bugs to synthetic biology to fuels. Curr Opin Biotechnol 2008, 19(6):556-63.

2. Ro DK, Paradise EM, Ouellet M, Fisher KJ, Newman KL, Ndungu JM, Ho KA, Eachus RA, Ham TS, Kirby J, Chang MCY, Withers ST, Shiba Y, Sarpong R, Keasling JD: Production of the antimalarial drug precursor artemisinic acid in engineered yeast. Nature 2006, 440(7086):940-943.

3. Levskaya A, Chevalier AA, Tabor JJ, Simpson ZB, Lavery LA, Levy M, Davidson EA, Scouras A, Ellington AD, Marcotte EM, Voigt CA: Synthetic biology: engineering Escherichia coli to see light. Nature 2005, 438(7067):441-442.

4. Tecon R, Wells M, Meer JR van der: A new green fluorescent proteinbased bacterial biosensor for analysing phenanthrene fluxes. Environ Microbiol 2006, 8(4):697-708.

5. Balagadde FK, Song H, Ozaki J, Collins CH, Barnet M, Arnold FH, Quake SR, You L: A synthetic Escherichia coli predator-prey ecosystem. Mol Syst Biol 2008, 4:187.
6. Cox RS, Surette MG, Elowitz MB: Programming gene expression with combinatorial promoters. Mol Systems Bio/ 2007, 3:145.

7. Alper H, Fischer C, Nevoigt E, Stephanopoulos G: Tuning genetic control through promoter engineering. Proc Natl Acad Sci USA 2005 102(36):12678-83.

8. Gardner TS, Cantor CR, Collins JJ: Construction of a genetic toggle switch in Escherichia coli. Nature 2000, 403(6767):339-342.

9. Basu S, Mehreja R, Thiberge S, Chen MT, Weiss R: Spatiotemporal control of gene expression with pulse-generating networks. Proc Natl Acad Sci USA 2004, 101(17):6355-6360.

10. Elowitz MB, Leibler S: A synthetic oscillatory network of transcriptional regulators. Nature 2000, 403(6767):335-338.

11. Win MN, Smolke CD: Higher-order cellular information processing with synthetic RNA devices. Science 2008, 322(5900):456-460

12. Benner SA, Sismour AM: Synthetic biology. Nat Rev Genet 2005 6(7):533-543.

13. Alon U: Biological networks: the tinkerer as an engineer. Science 2003 301(5641):1866-1867.

14. Andrianantoandro E, Basu S, Karig DK, Weiss R: Synthetic biology: new engineering rules for an emerging discipline. Mol Syst Biol 2006, 2 . 20060028

15. Bhalerao K: Synthetic gene networks: The next wave in biotechnology? Trends Biotechnol 2009, 27(6):368-74.

16. Maeda YT, Sano M: Regulatory dynamics of synthetic gene networks with positive feedback. J of Mol Biol 2006, 359(4):1107-1124.

17. Becskei A, Seraphin B, Serrano L: Positive feedback in eukaryotic gene networks: cell differentiation by graded to binary response conversion. The EMBO journal 2001, 20(10):2528-2535.

18. Williams JW, Cui X, Levchenko A, Stevens AM: Robust and sensitive control of a quorum-sensing circuit by two interlocked feedback loops. Mol Syst Biol 2008, 4:234

19. Nistala GJ, Wu K, Rao CV, Bhalerao KD: A modular positive feedbackbased gene amplifier. J Biol Eng 2010, 4:4

20. Wallin $\mathrm{E}$, von Heijne $\mathrm{G}$ : Genome-wide analysis of integral membrane proteins from eubacterial, archaean, and eukaryotic organisms. Protein Sci 1998, 7(4):1029-1038.

21. Klabunde T, Hessler G: Drug design strategies for targeting G-proteincoupled receptors. Chembiochem 2002, 3(10):928-944.

22. Lundstrom K: Structural genomics on membrane proteins: mini review. Comb Chem High Throughput Screen 2004, 7(5):431-439.

23. Wagner S, Klepsch MM, Schlegel S, Appel A, Draheim R, Tarry M, Högbom M, van Wijk KJ, Slotboom DJ, Persson JO, de Gier JW: Tuning Escherichia coli for membrane protein overexpression. Proc Natl Acad Sci USA 2008, 105(38):14371-6.

24. Rice CW, Hempfling WP: Oxygen-limited continuous culture and respiratory energy conservation in Escherichia coli. J Bacterio/ 1978 134:115-124.

25. Minghetti KC, Gennis RB: The two terminal oxidases of the aerobic respiratory chain of Escherichia coli each yield water and not peroxide as a final product. Biochem Biophys Res Commun 1988, 155:243-248.

26. Lorence RM, Gennis RB: Spectroscopic and quantitative analysis of the oxygenated and peroxy states of the purified cytochrome $\mathrm{d}$ complex of Escherichia coli. J Biol Chem 1989, 264(13):7135-7140.

27. Poellinger KA, Lee JP, JVP Jr, Greenberg EP: Intragenic suppression of a luxR mutation: characterization of an autoinducer-independent LuxR. FEMS microbiology letters 1995, 129:97-101.

28. Lutz $\mathrm{R}, \mathrm{Bujard} \mathrm{H}$ : Independent and tight regulation of transcriptional units in Escherichia coli via the LacR/O, the TetR/O and AraC/I1-12 regulatory elements. Nucleic acids research 1997, 15;25(6):1203-10.

29. Borisov V, Arutyunyan AM, Osborne JP, Gennis RB, Konstantinov AA Magnetic circular dichroism used to examine the interaction of Escherichia coli cytochrome bd with ligands. Biochemistry 1999 38(2):740-750.

doi: $10.1186 / 1754-1611-4-6$

Cite this article as: Bansal et al., A positive feedback-based gene circuit to increase the production of a membrane protein Journal of Biological Engineering $2010,4: 6$ 